1632 f. 1964, Presses Académiques Européennes, Bruxelles.

3. A. Ghouila-Houri, Caractérisation des matrices totalement unimodulaires, C. R. Acad. Sci. Paris 254 (1962), 1192-1194.

4. I. Heller, On linear systems with integralvalued solutions, Pacific J. Math. 7 (1957), 1351-1364.

5. W. Tutte, Matroids and graphs, Trans. Amer. Math. Soc. 90 (1959), 527-552.

6. P. Camion, Caractérisation des mutrices unimodulaires, Cahiers Centre Etudes Rech. 5 (1963), no. 4.

EURATOM, ISPRA, ITALY

\title{
PRIME RINGS WITH MAXIMAL ANNIHILATOR AND MAXIMAL COMPLEMENT RIGHT IDEALS ${ }^{1}$
}

KWANGIL KOH AND A. C. MEWBORN

1. Introduction. Let $R$ be a prime ring with a maximal annihilator right ideal and a maximal complement right ideal. Then there is a division ring $D$ such that either $R$ is isomorphic to a right order in the complete ring of linear transformations of a finite dimensional $D$-space, or for each positive integer $n$ there is a subring $R^{(n)}$ of $R$ which is isomorphic to a right order in the complete ring of linear transformations of an $n$-dimensional $D$-space. This is related to a result of $\mathrm{N}$. Jacobson $[2$, p. 33] and extends a theorem of A. W. Goldie $[1$; Theorem 4.4$]$ that a prime ring with maximum conditions on annihilator right ideals and complement right ideals is a right order in a simple ring with minimum condition on right ideals. $R$ is also isomorphic to a weakly transitive ring of linear transformations of a vector space. This is a generalization of a theorem of $\mathrm{R}$. E. Johnson $[4 ; 3.3]$.

2. We assume throughout that $R$ is a prime ring. The notation $R_{r}^{\Delta}\left(R_{l}^{\Delta}\right)$ is used to denote the right (left) singular ideal of $R$, and $L_{r}^{*}\left(L_{l}^{*}\right)$ is the lattice of closed right (left) ideals of $R$. An $R$-module is uniform if each pair of nonzero submodules has nonzero intersection. A right (left) ideal of $R$ is uniform if it is uniform as right (left) $R$-module. For other definitions and notation see [6].

THEOREM 1. $R$ contains a maximal annihilator right ideal and $a$ maximal complement right ideal if and only if $R_{r}^{\Delta}=(0)$ and $L_{r}^{*}$ is atomic.

Received by the editors July 1, 1964.

1 The authors wish to thank Professor R. E. Johnson for many helpful comments in revising the original manuscript of this paper. 
Proof. Assume first that $R$ contains a maximal annihilator right ideal $x^{r}, x \in R, x \neq 0$. Suppose $R_{r}^{\Delta} \neq(0)$. Then $R_{r}^{\Delta} x \neq(0)$; so there exists $y \in R_{r}^{\Delta}$ such that $y x \neq 0$. Now $x R \neq(0)$, so $y^{r} \cap x R \neq(0)$. If $x r \in y^{r} \cap x R$, $x r \neq 0$, then $r \in(y x)^{r}$ and $x^{r} \subset(y x)^{r}, x^{r} \neq(y x)^{r}$, a contradiction. Hence $R_{+}^{\Delta}=(0)$. If $R$ contains a maximal complement $C$, then a complement $A$ of $C$ is an atom of $L_{r}^{*}$. If $B \in L_{r}^{*}$, then $B A \neq(0)$ and $b A \neq(0)$ for some $b \in B . b A$ is an atom of $L_{r}^{*}$ contained in $B$. Hence $L_{r}^{*}$ is atomic.

Conversely, assume that $R_{r}^{\Delta}=(0)$ and $L_{r}^{*}$ is atomic. Let $A$ be an atom of $L_{r}^{*}$ and $a \in A, a \neq 0$. Then $a^{r}$ is a maximal annihilator right ideal and a maximal complement.

LеммA. Let $V$ be a uniform quasi-injective $R$-module with zero singular submodule and let $D=\operatorname{Hom}_{R}(V, V)$. If $v \in V$, then $D v$ has nonzero intersection with each nonzero submodule if and only if there is a nonzero element $r \in R$ such that $r \cdot v^{r}=(0)$.

Proof. Let $v^{r l}=\left\{r \in R: r \cdot v^{r}=(0)\right\}$ and suppose $v^{r l} \neq(0)$. Let $V_{1}$ be a nonzero submodule of $V$. Let $w \in V_{1}, w \neq 0$. Then $w \cdot v^{r l} \neq(0)$. Let $r \in v^{r l}$, wr $\neq 0$. Then $v^{r} \subseteq r^{r} \subseteq(w r)^{r}$. By $[6,2.2], v$ and wr generate the same 1-dimensional $D$-subspace of $V$. Hence $D v \cap V_{1} \neq(0)$.

Conversely, suppose that $D v$ has nonzero intersection with each nonzero submodule of $V$. There is a nonzero right ideal $I$ of $R$ such that $v^{r} \cap I=(0) . v I \cap D v \neq(0)$. Let $i \in I, d \in D$ be such that $v i=d v \neq 0$. Then $v^{r}=(d v)^{r}=(v i)^{r}=i^{r}$. Thus $i \in v^{r l}$ and $v^{r l} \neq(0)$.

Theorem 2. Let $R$ be a prime ring for which $R_{r}^{\Delta}=(0)$ and $L_{r}^{*}$ is atomic. Then there is a division ring $D$ such that (1) $R$ is a right order in the ring of all linear transformations of a finite dimensional $D$-space, or (2) for each positive integer $n$ there is a subring $R^{(n)}$ of $R$ such that $R^{(n)}$ is a right order in the ring of all linear transformations of an $n$ dimensional D-space.

Proof. The maximal right quotient ring $R^{\prime}$ of $R$ is primitive with minimal right ideal $I^{\prime}$. We first show that $I^{\prime}$ is a quasi-injective $R$ module. ${ }^{2}$ Let $U$ be a nonzero $R$-submodule of $I^{\prime}$ and suppose $\alpha$ is a nonzero homomorphism of $U$ into $I^{\prime}$. Let $u \in U$ such that $\alpha(u) \neq 0$. $R^{\prime \Delta}=(0)$ because $R_{T}^{\Delta}=(0)$. Thus there is a nonzero right ideal $J^{\prime}$ of $R^{\prime}$ such that $\left\{r^{\prime} \in R^{\prime}: u r^{\prime}=0\right\} \cap J^{\prime}=(0)$. Define $\tilde{\alpha}$ on $I^{\prime}=u J^{\prime}$ by: $\tilde{\alpha}\left(u r^{\prime}\right)=\alpha(u) r^{\prime}, r^{\prime} \in J^{\prime} . \quad \tilde{\alpha} \in \operatorname{Hom}_{R}\left(I^{\prime}, I^{\prime}\right)$. Suppose $v \in U, v=u r^{\prime}$, $r^{\prime} \in J^{\prime}$. Then $K=\left\{r \in R: r^{\prime} r \in R\right\}$ is a large right ideal of $R$. If $r \in K$, then $\alpha(v) r=\alpha(v r)=\alpha\left(u r^{\prime} r\right)=\alpha(u) r^{\prime} r$; hence $r \in\left(\alpha(v)-\alpha(u) r^{\prime}\right) r$. Since

\footnotetext{
2 This fact is a consequence of the following unpublished result of $R$. E. Johnson: If $R_{r}^{\Delta}=(0)$ and $R \subset S \subset R^{\prime}$, and if $M$ is a quasi-injective $S$-module, then $M$ is a quasi-injective $R$-module.
} 
$K$ is large and $R_{r}^{\Delta}=(0)$, we conclude $\alpha(v)=\alpha(u) r^{\prime}=\tilde{\alpha}\left(u r^{\prime}\right)=\tilde{\alpha}(v)$. This proves that $\tilde{\alpha}$ is an extension of $\alpha$, and we conclude that $I^{\prime}$ is a quasiinjective $R$-module.

Now, let $I=I^{\prime} \cap R$. $I$ is a uniform $R$-module with zero singular submodule. Let $D=\operatorname{Hom}_{R}\left(I^{\prime}, I^{\prime}\right)=\operatorname{Hom}_{R^{\prime}}\left(I^{\prime}, I^{\prime}\right)$. Then $D$ is a division ring and $V=D I$ is a minimal quasi-injective extension of $I$. $D$ $=\operatorname{Hom}_{R}(V, V)$. Let $B$ be a basis for $V$ as (left) vector space over $D$. If $B$ is finite, then $L_{r}^{*}$ is finite dimensional $[6,3.1]$. Hence, in this case, $R$ is a right order in a simple ring with minimum condition on right ideals $[1,4.4]$.

Suppose $B$ is not finite and let $n$ be a positive integer. Let $V_{1}$ be the subspace of $V$ spanned by a subset $\left\{v_{i}\right\}_{i=1}^{n}$ of $B$. Choose $e \in R^{\prime}$ such that $v e=v$, each $v \in V_{1}$, and such that $e$ has rank $n$ as linear transformation of $V$. Let $R^{(n)}=\left\{r \in R \cap e R^{\prime}: V_{1} r \subseteq V_{1}\right\} . R^{(n)}$ is a subring of $R$ isomorphic to a subring of the full ring $Q$ of linear transformations of $V_{1}$. We show that $R^{(n)}$ is a right order in $Q$. Let $q \in Q$ of rank $k \geqq 1$. Let $\left\{w_{i}\right\}_{i=1}^{n}$ be a basis for $V_{1}$, and if $k<n$ we assume that this basis is chosen so that $\left\{w_{i}\right\}_{i=k+1}^{n}$ is a basis for the null space of $q$. Let $\left\{u_{i}\right\}_{i=1}^{n}$ be a basis for $V_{1}$ chosen so that $u_{i}=w_{i} q, 1 \leqq i \leqq k$. If $J_{i}=\bigcap_{j \neq i} w_{j}^{r}$ and $K_{i}=\bigcap_{j \neq i} u_{j}^{r}$, then $w_{i} J_{i} \neq(0)$ and $u_{i} K_{i} \neq(0), 1 \leqq i \leqq n$, by $[6,2.2]$. Let $r_{i} \in J_{i}$ and $s_{i} \in K_{i}$ such that $w_{i} r_{i} \neq 0, u_{i} s_{i} \neq 0,1 \leqq i \leqq n$. Then $T=\left\{r \in R: e r_{i} r \in R, e s_{i} r \in R, 1 \leqq i \leqq n\right\}$ is a large right ideal of $R$. By the Lemma and the fact that $R_{r}^{\Delta}=(0), w_{i} r_{i} T \cap u_{i} s_{i} T \cap D v_{i} \neq(0)$. Let $a_{i} \in T, b_{i} \in T$ such that $w_{i} r_{i} a_{i}=u_{i} s_{i} b_{i}$ is a nonzero element of $D v_{i}$. If $r=\sum_{j=1}^{k} r_{j} a_{j}$ and $s=\sum_{j=1}^{n} s_{j} b_{j}$, then $e r$ and $e s$ are in $R^{(n)}$ and $w_{i} q e s$ $=w_{i} e r, 1 \leqq i \leqq n$. Moreover, es is nonsingular on $V_{1}$. This proves that $R^{(n)}$ is a right order in $Q$.

COROLlARY. $R$ contains a subring which is a right order in a division ring.

THEOREM 3. Let $R$ be a prime ring such that $R_{r}^{\Delta}=(0)$ and $L_{r}^{*}$ is atomic. Let $U$ be an atom of $L_{r}^{*}$ and $V$ the minimal quasi-injective extension of $U$ as right $R$-module. If $D=\operatorname{Hom}_{R}(V, V)$ there exists a subring $K$ of $R$ which is a right order in $D$ and such that if $\left\{v_{i}\right\}_{i=1}^{n}$ is a finite $D$-linearly independent subset of $U$ and $\left\{y_{i}\right\}_{i=1}^{n}$ is a sequence in $U$, then there exists $r \in R$ and $k \in K, k \neq 0$, such that $v_{i} r=k y_{i}, 1 \leqq i \leqq n$.

Proof. Let $R^{\prime}, I^{\prime}$, and $V$ be chosen as in the proof of Theorem 2. There exists $u \in U$ such that $u^{r} \cap I^{\prime}=(0)$. Then $u I^{\prime}=I^{\prime}$. Let $e \in I^{\prime}$ be such that $u e=u$. Then $e^{2}=e$ and $e R^{\prime} e$ is a division ring isomorphic to $D$. Let $L=R^{\prime} e \cap R$, and let $K=e R^{\prime} e \cap R=\left(e R^{\prime} \cap R\right) \cap\left(R^{\prime} e \cap R\right)$ $=U \cap L . L$ is a left ideal of $R$ and $K$ is a subring of $R$ and $e R^{\prime} e$. Let 
$d=e r^{\prime} e, r^{\prime} \in R^{\prime}, d \neq 0$. Then $d U \cap U \neq(0)$. Let $I=\{u \in U: d u \in U\}$. Then $I \neq(0)$ and $I \cap L \neq(0)$. If $k_{1} \in I \cap L, k_{1} \neq 0$, then $d k_{1} \in K$. This proves that $K$ is a right order in $e R^{\prime} e$.

Let $\left\{v_{i}\right\}_{i=1}^{n}$ be a finite $D$-linearly independent subset of $U$ and $\left\{y_{i}\right\}_{i=1}^{n}$ a sequence in $U$. For $1 \leqq i \leqq n$, let $J_{i}=\bigcap_{j \neq i} v_{j}$. By $[6,2.2]$ $v_{i} J_{i} \neq(0)$. Let $k \in K, \vec{k} \neq 0$. Then $v_{i} J_{i} \vec{k} \neq(0)$. Select $b_{i} \in J_{i}$ such that $v_{i} b_{i} \bar{k} \neq 0$. Then $v_{i} b_{i} \bar{k} \in K$ and $\bigcap_{i=1}^{n}\left(v_{i} b_{i} \bar{k}\right) K \neq(0)$. Hence $v_{i} b_{i} \tilde{k} k_{i}=k \neq 0$, $1 \leqq i \leqq n$, for some $k_{i}, k$ in $K$. If $r=\sum_{i=1}^{n} b_{i} \bar{k}_{i} y_{i}$, then $v_{i} r=k y_{i}$, $1 \leqq i \leqq n$. The theorem is proved.

Suppose now that we also assume in Theorem 3 that $R_{l}^{\Delta}=(0)$ and $L_{l}^{*}$ is atomic. Then we can show that $K$, (and hence $L$ ) is also left uniform. For assume $K_{1}$ and $K_{2}$ are nonzero left ideals of $K$ and that $K_{1} \cap K_{2}=(0)$. If $k \in K, k \neq 0$, then $K_{1} k \cap K_{2} k=(0)$. But $(L k)^{*}$ is an atom of $L_{l}^{*}$ by $[4,1.1]$. If $0 \neq k_{1} \in K_{1}$ and $0 \neq k_{2} \in K_{2}$, then $L k_{1} k \cap L k_{2} k$ $\neq(0)$. Suppose $l_{1} k_{1} k=l_{2} k_{2} k \neq 0$. Let $k^{\prime} \in U$ such that $k^{\prime} l_{1} k_{1} k=k^{\prime} l_{2} k_{2} k$ $\neq 0$. Then $k^{\prime} l_{1} k_{1} \in K_{1}$ and $k^{\prime} l_{2} k_{2} \in K_{2}$, and so $K_{1} k \cap K_{2} k \neq(0)$, a contradiction. Clearly $L$ is uniform. Since $K$ is left uniform it is a left order in $D$. This implies that a $K$-linearly independent subset of $U$ is also $D$-linearly independent. This shows that $[4,3.3]$ is a consequence of Theorem 3.

\section{BIBLIOGRAPHY}

1. A. W. Goldie, Semi-prime rings with maximum condition, Proc. London Math Soc. 10 (1960), 201-220.

2. N. Jacobson, Structure of rings, Amer. Math. Soc. Colloq. Publ. Vol. 37, Amer. Math Soc., Providence, R. I., 1956.

3. R. E. Johnson, The extended centralizer of a ring over a module, Proc. Amer. Math. Soc. 2 (1951), 891-895.

4. - Representations of prime rings, Trans. Amer. Math. Soc. 74 (1953), $351-357$.

5. - Quotient rings of rings with zero singular ideal, Pacific. J. Math. 11 (1961), 1385-1392.

6. R. E. Johnson and E. T. Wong, Quasi-injective modules and irreducible rings, J. London Math. Soc. 36 (1961), 260-268.

University of North Carolina 